\title{
Computational Intelligence and Voltage Stability Analysis for Mitigation of Blackout
}

\author{
Fouzul Azim Shaikh \\ EN department \\ KIET, Ghaziabad \\ U.P., India, 201206
}

\author{
Dr Zaheeruddin \\ EE Department \\ JMI, New Delhi \\ India, 110025
}

\author{
Dr M S Jamil Asghar \\ EE Department \\ AMU, Aligarh, U.P. \\ India, 202002
}

\begin{abstract}
Mitigation of power system blackouts are still going on all over the world. Millions of people are affected each year due to power system blackouts. Integration the knowledge of computational intelligence with roots of blackout can lead us to triumph over these tremendous losses. Voltage stability study is an essential aspect as it avoids power system blackouts. This paper presents a computational intelligence based evaluation for a very important aspect, voltage stability of an electric power system. Large amounts of data, fuzziness of that data, and the endless variations of system configurations are all factors contributing to the complexity of power system analysis and diagnosis. This complexity has necessitated the need for computational intelligent tools to aid system engineers. Artificial Neural Network and Fuzzy Logic have emerged as amongst most suitable tools for power system applications. The combination of two intelligent disciplines into one system i.e. neuro-fuzzy technique for power system analysis has proven even more effective.
\end{abstract}

\section{Keywords}

Computational intelligence, power system blackout, neural network.

\section{INTRODUCTION}

Computational intelligence has acquired intensified interest and importance during the last decade for many reasons like blackouts of Washington, Tokyo, England, Sweden and almost every big or small power system. Power system blackout has become an issue of great concern for utility companies, for their customers, and for electric equipment manufacturers, operation and reliability. The proper diagnosis of blackout problems requires a high level of engineering expertise and the required expert knowledge with computational intelligence. Powerful tools of interest to power system diagnosis are those of artificial neural networks (ANN), fuzzy logic (FL), and the newer techniques of adaptive neuro-fuzzy systems (ANFS). These tools will be introduced and defined. The problems involved in performing power system disturbance diagnosis will also be exposed. The interest in blackout involves all three parties concerned with the power business: utility companies, equipment manufacturers, and electric power customers. There are many reasons for the growing concern with blackouts. Voltage stability is the capability of a power system to maintain steady tolerable voltages at all the buses in the system under normal operating conditions and after being subjected to a disturbance [3]. The major types of power system disturbances diagnostics are [1, 2]: Impulsive transients, Repetitive impulsive transients, under voltages, Short interruptions, Voltage swells (surges), DC offset (distortion), Inter harmonics (distortion), Voltage fluctuation (flicker), Power frequency variations, Oscillatory transients, Over voltages, Sustained interruptions, Voltage sags (dips), Voltage imbalance, Harmonics (distortion), Power frequency variations. Some characteristics and indices used by experts to diagnose power system disturbances problems may be summarized in the following: Maximum (peak) voltage level of disturbance (impulse), Phase angle placement of the event on the sine wave, Voltage magnitude, Rate of rise, Rate of decay, Duration of event, Frequency spectrum, Dominant harmonic, Decay time, Frequency of occurrence, Percentage odd/even harmonics, total harmonic distortion (THD).

During the past fifteen years, there has been a continually increasing interest and investigation into voltage instability and collapse. The first paper related to voltage instability appeared in 1968 by Weedy and Cox on Voltage stability of radial power links. Venikov proposed the first criteria for detecting the point of voltage collapse in 1975, on Estimation of electric power system steady state stability in load flow calculation. Active work involving voltage stability started in 80 's. Predicting the voltage stability index of a large-scale power system using artificial neural network is presented by M. Moghavvemi [8]. It has been shown that ANN can be used as an operator aid in monitoring the level of stability of the power system. It was further demonstrated that more accurate results can be obtained if the large power system is sectionalized into smaller subsystems making it possible to use smaller ANN for prediction of the stability level of the power system. Gubina [9] determined voltage collapse proximity index using voltage phasor approach. An adequate proximity index is calculated based on the voltage phasor values only. Furthermore, an algorithm is derived which automatically detects the network transmission paths to the load nodes, which are prone to voltage collapse due to additional real or reactive loading. Brucoli presented a method for analyzing the voltage stability problem in electric power system. From linearized model in state space 
form of a multi machine power system the voltage stability conditions are derived on the basis of suitable model of the original system capable of retaining the dynamics of voltage at generator and load nodes. The approach allows a systematic individualization of all the dynamic factors that affect voltage instability phenomena and suggests the appropriate representation that has to be adopted for each dynamic component of the system. Price [10] considered the global response of a power system to variation in the node constraints. A generalized circle diagram approach for global analysis of transmission system performance presented. The relation of specific system variables to independent node parameters is calculated using a numerical technique that provides a generalization of the elementary power circle diagram. Curves that show system performance during disturbances, tradeoffs between design parameters, voltage stability limits and multiple power flow solutions are produced node parameter. Repo [11] presented the use of neural network for voltage stability by calculation of margins by the point of collapse method. El-Keib [12] investigated the application of ANN in voltage stability assessment. Based on the energy methods, a direct mapping relation between system loading condition and the voltage stability margins is set up via the ANN. A systematic method for selecting the ANN input variables are also developed using sensitivity analysis. Moon et al [13] have shown that all major methods used in static voltage stability analysis, i.e. the jacobian method, voltage sensitivity method, real and reactive power loss sensitivity methods and energy function methods have an identical background in theory. Abe et al [14] introduced first order delay model of a load admittance changes. Then, using this model, a set of linearized dynamic equation is derived and stability conditions are obtained. Hill [15] proposed a simple dynamic model, which captures the usual nonlinear steady-state behavior plus load recovery and overshoot. The parameters of the model can be related to physical devices depending on the time zone following a disturbance. Simple dynamic voltage stability analysis is developed based on the model. Goa et. al. [16] discussed the voltage stability analysis using a modal analysis technique. The method computes, using a steady state system model, a specified number of the smallest eigenvalues and the associated eigenvectors of a reduced jacobian matrix. The eigenvalues, each of which is associated with a mode of voltage/reactive power variation, provide a relative measure of proximity to voltage instability.

A system cross the threshold in the state of voltage instability when a disorder, increase in load demand, or change in system condition causes progressive and unmanageable drop in voltage. The main factor causing instability is the inability of the power system to meet the demand of reactive power. The sensitivity of the problem is usually the voltage drop that occurs when active power and reactive power flow through inductive reactance associated with the transmission network.

\section{VOLTAGE STABILITY}

A measure for voltage stability is that, at a given operating condition for every bus in the system, the voltage magnitude increases as the reactive power insertion at the same bus is increased. A system is voltage unstable if, for at least one bus in the system, the bus voltage magnitude (V) decreases as the reactive power injection $(\mathrm{Q})$ at the same bus is increased. Major methods for static voltage analysis are: Jacobian method, Voltage sensitivity method, Real and reactive power loss sensitivity method. (i) Jacobian Method: Voltage instability is due to the structural instability of the system resulting from change of system parameters. In some special cases, parameter changes in the system state transfer to the saddle node. If this happens, small variations of the parameters can make the system state branch off into two kinds of nodes, i.e., stable nodes and unstable nodes. The Jacobian method shows the condition of saddle node bifurcation by examining the power flow solutions with the changes in the bus injection powers. On the basis of the jacobian method, the determinant or the least absolute value of the eigen values is sometimes utilized as a performance index of voltage collapse. (ii) Voltage sensitivity method: The voltage sensitivity method is the most direct approach using the voltage sensitivity to the system parameters. The voltage sensitivity goes to infinity at the collapse point. This collapse condition should be identical with that of the jacobian method. (iii) Power Loss Sensitivity Method: The voltage collapse phenomenon is accompanied by the rapid increase in line flows. At the collapse point, the losses increasing ratios dPloss/dP, dQloss/dQ, dQloss/dP, dQloss/dQ go to infinity. This fact provides the theoretical background for the power loss sensitivity method.

In order to keep the consistency in voltage stability analysis, this condition should agree with the collapse condition of the Jacobian method that the Jacobian matrix is singular at the voltage collapse point. These are two broad types of tools for voltage stability analysis: dynamic and static. Dynamic analysis uses time-domain simulations to solve non-linear system differential and algebraic equations. Static analysis is based on the solution of conventional or modified power flow equations. Dynamic analysis provides very accurate replication of the time responses of the system. However, the dynamic simulation consumes a lot of computer time and also does not provide information regarding the sensitivity or degree of instability. On the other hand, static analysis is ideal for the bulk of studies in which voltage stability limits for many pre-contingency and post-contingency cases must be determined. It involves only the solution of algebraic equations and therefore is computationally more efficient. The only feasible way to effectively plan the voltage stability of a system is to use both the static and dynamic analysis programs in a complimentary manner. 


\section{COMPUTAIONAL INTELLIGENCE}

\subsection{Artificial Neural Networks}

Artificial neural networks (ANN) can be defined as being very simple arithmetic computing units (nodes) connected together in a highly parallel network of interconnected layers, and as being able with this kind of structure to represent functions and to learn these functions from examples. The one ELEMENT, or NODE "i" of an ANN have number of inputs "aj" entering the unit via "links". Each link has a weight "w" associated with it. Inside the unit there is the input function which calculates the input to the unit as the summation of the individual inputs, each multiplied by the weight of the link connecting it to unit "i". The summed input is operated on by an activation function "g". The result of this operation is the output of this unit "ai", which is a single output value propagated to the next layer of units. A multilayer feed-forward ANN is characterized by having one or more layers of neurons other than the input and output layers, known as hidden layers. It is also characterized by the onedirectional flow of data between layers. The knowledge represented and stored in the ANN is represented in the weights associated with each link. The learning process of ANN is termed the "training" of the network. Back-propagation is the most popular technique used for training ANN. In backpropagation, after deciding on certain architecture for the network (i.e., number of layers, units per layer, and activation functions), preliminary weights are assigned throughout the network. The inputs of the training cases (examples of the function to be learned where both input and output are known) are applied to the network and the error between the actual output and required output is calculated. This error is then used to modify the weights related to the network links. The training cases are re-passed to the network with the new adjusted weights and the error is recalculated. The process is iterative and terminates when either a satisfactory level of error is achieved or when a preset number of iterations are reached. It should be noted that there are no guidelines for the choice of network architecture except for the experience of the user and a trial and error mechanism. However, it must be taken into consideration that the more complex the ANN the longer the training times required, and the higher the probability of error divergence.

ANNs are never 100 percent correct, but, in practical applications, it is usually better to have a solution, which is 99 percent correct in a few milliseconds, rather than have a 100 percent correct solution in tens of hours.

\subsection{Applications of ANN and Results}

The proposed method has been tested using a 5-bus system shown in Fig. 1. The system has two generators on bus 1 and 2, with loads on buses 1, 2, 3 and 5. One switched shunt at bus 3 . Total actual load for in-service devices only is 500.00 MW and generation is 505.4 MW. Losses are 5.40 Mw. Load demand
Mvar is 150.0 and generated Mvar is 170.0. Positive generator spinning reserves are 894.6 MW and negative spinning reserves are 355.40 MW.

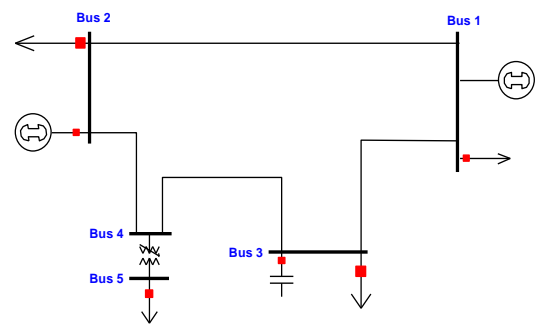

Fig. 1. Sample power system

For different sets of input parameters, simulations are performed to generate the necessary training data. For each distinct operating condition, the corresponding load and shunt values recorded. The input/output training patterns used for the learning phase of the ANN are given in Table-1. Nine patterns are used for training the network. The switchable shunt is kept on bus 3 and firstly kept off, while load is increased at bus 3. All data are in per unit on a base of 100 MVA. The learning data input/output patterns are presented to the ANN during the learning phase. Commercially available ANN toolbox from MATLAB ${ }^{\circledR}$, implementing the back propagation method, is used. The neural network has 9 inputs, 15 hidden neurons and 9 outputs.

Table 1. Value of Mvar needed at to avoid voltage collapse

\begin{tabular}{|c|c|c|}
\hline S.No. & $\begin{array}{c}\text { Active power } \\
(\mathrm{MW}) / 100\end{array}$ & Actual Mvar needed \\
\hline 1 & 7.01 & 0.0 \\
\hline 2 & 7.02 & 2.0 \\
\hline 3 & 7.03 & 3.2 \\
\hline 4 & 7.04 & 4.5 \\
\hline 5 & 7.05 & 5.7 \\
\hline 6 & 7.06 & 7.6 \\
\hline 7 & 7.08 & 10.2 \\
\hline 8 & 7.10 & 12.9 \\
\hline 9 & 7.12 & \\
\hline
\end{tabular}




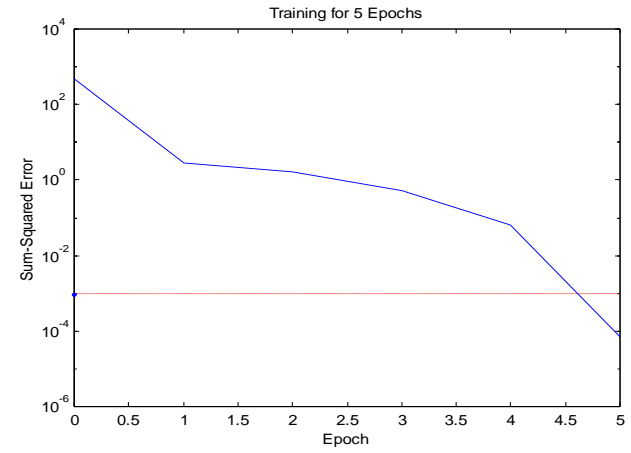

Fig.2. Sum-squared error obtained for training of ANN at shunt support

It is obvious from the output vector in Table-I that for the voltage stable point the target is Mvar values is zero at beginning up to $700 \mathrm{MW}$. Continuous increase from $701 \mathrm{MW}$ to 702 MW of load; a point comes where voltage blackout for complete power system occurs. When the shunt is kept on at bus-3 the collapse at loading factor 7.01, giving normal operation, but on the verge of collapse. This point of collapse is a shifted by the insertion of a switched shunt at the bus 3 .

Table-2 shows the ANN results of actual Mvar needed to avoid voltage collapse, obtained from trained neural networks. Seven input values of load are taken for this analysis and corresponding output compared with actual results in Figure 3.

Table 2. ANN results for actual Mvar needed to avoid voltage collapse

\begin{tabular}{|c|c|c|}
\hline $\begin{array}{c}\text { S. } \\
\text { No. }\end{array}$ & $\begin{array}{c}\text { Active power } \\
(\mathrm{MW}) / 100\end{array}$ & $\begin{array}{c}\text { Actual Mvar } \\
\text { needed }\end{array}$ \\
\hline 1 & 7.015 & 00.5229 \\
\hline 2 & 7.025 & 01.1450 \\
\hline 3 & 7.055 & 05.3805 \\
\hline 4 & 7.075 & 07.1717 \\
\hline 5 & 7.090 & 09.0187 \\
\hline 6 & 7.110 & 12.4275 \\
\hline 7 & 7.130 & 12.9100 \\
\hline
\end{tabular}

Comparison between ANN and actual results are shown in Fig. 3. Shunt Mvar needed at bus 3 to avoid collapse kept on X-axis with MW values of load in multiplication of $100 \mathrm{MW}$ are taken on the $\mathrm{Y}$-axis is taken for critical point of voltage collapse. ANN results, which solved by ANN toolbox available on MATLAB from math works giving almost coinciding results for the value obtained by conventional methods.

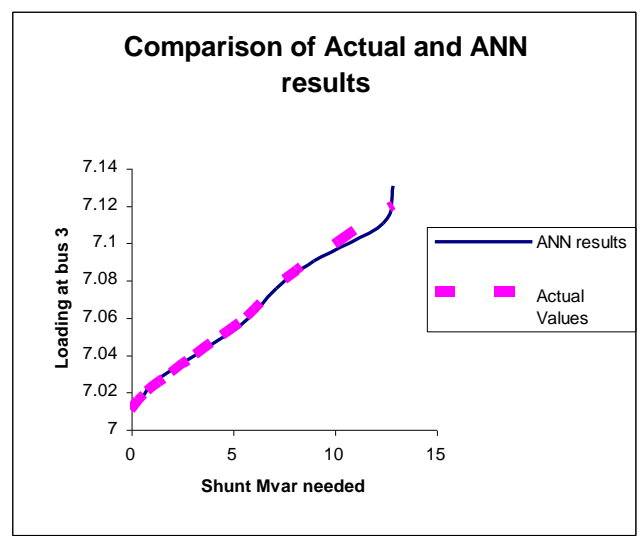

Fig. 3. Comparison for $\mathrm{ANN}$ and actual results for different loadings

\subsection{Fuzzy Logic for Blackout Mitigation}

Fuzzy logic and fuzzy sets are tools for expressing and operating on knowledge that is imprecise, or where the interpretation is highly subjective and depends strongly on context or human opinion [4]. Crisp variables and crisp knowledge are element in the knowledge domain that has an exact truth value, either TRUE or FALSE. Considering the warmth of the weather, cold, warm, and hot are all terms to which no specific degree of temperature could be allocated. In fuzzy logic, fuzzy rules are used to express knowledge in terms of these imprecise ideas. For example:

IF the weather is WARM

AND the wind is LIGHT

THEN take off some of your clothing

Expressed in a conventional (crisp) rule it would be:

IF the temperature is greater than $70^{\circ} \mathrm{F}$

AND the wind speed is less than $5 \mathrm{mph}$

THEN take off your coat.

Not only is the fuzzy logic rule more natural and more expressive, but it will fire over a wider range of temperatures and wind speeds. Hence, one fuzzy logic rule can replace many conventional rules. The basic elements of a fuzzy model and the main operational processes of a fuzzy system can be summarized as follows: 
Knowledge base (KB) of fuzzy rules.

Fuzzy model, which is a group of fuzzy sets that model the system variables.

Fuzzifier converts the system's inputs into a fuzzy format.

Fuzzy inference system, which executes all the fired rules and generates a new fuzzy output set.

Defuzzifier, which converts the output fuzzy set into a crisp solution value.

A fuzzy set is a set of elements that have a degree of belonging or membership attached to each one. A fuzzy set has membership degrees from " 0 " to " 1 ." The system is considered modeled when fuzzy sets are created for all the system input variables, and also for the output variable, based on the opinion of an expert or engineer. The final stage in completing a fuzzy system is that, once a fuzzy model has been constructed, several simulations are performed, the results of which are compared to known test cases. If the results are not satisfactory, changes are made to either the fuzzy set descriptions (membership functions) or to the interactions encoded in the rules [5].

\section{The Role of Soft Computing}

In the last few years various blackouts all over the world creating worries in power engineering operators. Applicability of artificial intelligence is also shown in various papers for power engineering power operators [17]. As they expect that conventional methods be used at top level in America and England even then where they are lacking in power control techniques. So the need of Artificial Intelligence is a demand of power operators. Soft computing differs from conventional (hard) computing in that, unlike hard computing, it is tolerant of imprecision, uncertainty, partial truth, and approximation. In effect, the role model for soft computing is the human mind. Soft Computing (SC) is a group of methodologies, which provide a foundation for the conception, design, and deployment of intelligent systems. The principal members of SC are fuzzy logic (FL), neuro-computing (NC), genetic computing (GC) and probabilistic computing (PC), with PC subsuming evidential reasoning, management of uncertainty and parts of machine learning theory. Within SC, the main contribution of FL is a methodology for dealing with imprecision, approximate reasoning, fuzzy information granulation and computing with words; that of $\mathrm{NC}$ is system identification, learning and adaptation; that of GC is systematized random research, tuning and optimization: and that of PC is decision analysis and management of uncertainty. The essence of soft computing is that unlike the traditional, hard computing, soft computing is aimed at an accommodation with the regular imprecision of the real world, Thus, the guiding principle of soft computing is: Exploit the tolerance for imprecision, uncertainty and partial truth to achieve tractability, robustness, low solution cost and better relationship with reality. In the main, FL, NC, GC and PC are complementary rather than competitive. For this reason, it is frequently advantageous to use $\mathrm{FL}, \mathrm{NC}, \mathrm{GC}$ and $\mathrm{PC}$ in combination rather than exclusively, leading to so-called "hybrid intelligent systems." At this juncture, the most visible systems of this type are neuro-fuzzy systems. We are also beginning to see fuzzy-genetic, neuro-genetic and neuro-fuzzy-genetic systems.
Such systems are likely to become everywhere in the not distant future. In coming years, the presence of intelligent systems is certain to have a reflective impact on the ways in which manmade systems are conceived, designed, manufactured, employed and interacted with. This is the perspective in which the basic issues relating to Soft Computing Techniques and PowerWorld ${ }^{\circledR}$ Simulator for forecasting and mitigation of power system blackout may provide intelligent power system.

\section{Need of PowerWorld Simulator}

As power systems become increasingly complex, there is a critical need to make available improved tools for training power system engineers and analysts. Q-V curve interpretations of energy measures for voltage security also discussed already using PowerWorld simulator. Traditionally this training has been provided primarily to power operator engineers, system operators, and practicing engineers. Basic concepts needed to be presented simply, yet the program required sufficient detail to maintain the interest of and provide challenge to the advanced engineering power operator. The principal thrusts of the program include basic power flow in a network, how system controls (such as generator MW/voltage set point, LTC transformers, line outages, and power transactions) affect power flow, and area control concepts such as area control error (ACE), automatic generation control (AGC) and economic dispatch (ED). The use of animated power flow arrows also helps to convey how power flows from the generators through the transmission network to the loads. Also, tabular displays of many different quantities such as bus voltages and loads, generator outputs, and line flows could be used to supplement the one-line values. To better replicate actual system operation, in which power system operators and engineers work to insure that there are no violations in either the base case or for a set of statistically likely contingencies, the design project required the power operators to perform a full contingency analysis solution. If done manually this would have required the power operators to sequentially open each of the individual lines and transformers, solve the power flow, check for violations, and then close the device. A power operator can start operating just a two-bus system and then work up to increasingly larger systems. The one-line diagram and simulation time windows are then displayed along with a menu bar. At any time during the simulation, the power operator can interact with the case (such as by changing controls or setting up MW transactions) through the various windows. The one-line diagram is the most important part of the graphical user interface (GUI). The oneline representation, like all of the other windows in the program, has been designed using object-oriented techniques. The advantages of this approach include flexibility to allow the user to interact with all objects on the screen, small source and executable files, extensibility and reusability of existing code, and a user-friendly graphical user interface (GUI). The one-line uses graphical symbols to represent system equipment such as buses, generators, loads, transmission lines and transformers. Hence a power operator can easily design his own area system and can implement the Soft Computing Techniques and PowerWorld ${ }^{\circledR}$ Simulator for forecasting and mitigation of power system blackout. 


\section{CONCLUSION}

Exposing two of the hottest topics in the electric power industry, namely power system blackouts and computational intelligence, has been attempted. Voltage instability for power system has become an issue of great concern for utility companies, for their customers, and for electric equipment manufacturers, due to the negative impact that power disturbances have on system operation and reliability. Large amounts of data, fuzziness of that data, and the endless variations of system configurations are all factors contributing to the complexity of power quality analysis and diagnosis. This complexity has necessitated the need for sophisticated tools to aid system engineers. Artificial Neural Network and Fuzzy Logic have emerged as amongst most suitable tools for voltage stability for power system applications. The combination of two or more intelligent disciplines into one system for power system analysis has proven even more effective. ANFS applications in voltage stability for power blackouts are certainly a very valid alternative, one that still holds a lot of potential for future investigation and research.

\section{ACKNOWLEDGMENTS}

Our thanks to the HOD EN Department KIET, Professor G Singh, Director, Dr. O P Jain and KIET management for providing facilities and encouragements.

\section{REFERENCES}

[1] R.C. Dugan, M.F. McGranaghan, H.W. Beaty, Electric power systems quality, McGraw-Hill, 1996.

[2] The Dranetz field handbook for power quality analysis, Dranetz Technologies, 1991.

[3] P. Kundur, Power system stability and control, McGrawHill: New York 1993.

[4] E. Cox, "Fuzzy fundamentals," IEEE Spectrum, October 1992, pp. 58-61.

[5] T.J. Ross, Fuzzy logic with engineering applications, McGraw-Hill, 1995.

[6] J.S.R. Jang, C.T. Sun, E. Mizutani, Neuro-fuzzy and soft computing: a computational approach to learning and machine intelligence, Prentice-Hall, 1997.
[7] W.R. Anis, M.M. Morcos, "Electric power quality and artificial intelligence: applicability and overview," Proceedings of the 1998 Large Engineering Systems Conference on Power Engineering, Halifax, Nova Scotia, Canada, pp. 107-111.

[8] Brucoli M., Rossi F., Torelli F., Trovoto M., "A generalized Approach to the Analysis of Voltage Stability in Electric Power Systems", EPSR, 9(1985) 49-62.

[9] Salatino D., Sbizzai R., Trovato M., La Scala M., "Online Voltage Stability Assessment of Load Centers by Using Neural Networks", EPSR, 32 (1995).

[10] Price G.B., "A Generalized Circle Diagram Approach for Global Analysis of Transmission System Performance", IEEE Trans., Vol. PAS-103, No. 10, (October 1984), 28812890.

[11] S. Repo et.al. "Multilayer perceptron neural network based static voltage stability assessment", Technical reprt, TUT Power Engineering Tampere, Finland.

[12] A.A. El-Keib and X. Ma., "Application of Artificial Neural Networks in Voltage Stability Assessment," IEEE Transactions on Power Systems, Vol. 10, No. 4, November1995.

[13] Y.H. Moon and S. Kim, "On the identity of static voltage stability analysis methods in power systems", Int. J. of Power and Eng. Syst., vol. 19, No 1, 1999.

[14] S. Abe et.al. "Power system voltage stability", IEEE Trans. on Power Apparatus and Systems, Vol. PAS-101, No. 10 Oct. 1982.

[15] D. J. Hill, "Nonlinear dynamic load models with recovery for voltage stability studies", Publication 92WM 102-4 PWRS, Power Engineering Society.

[16] B. Gao et.al. "Voltage stability evaluation using modal analysis", IEEE Transactions on Power Systems, Vol. 7, No. 4, November 1992.

[17] Fouzul Azim Shaikh, M. S. Jameel Asghar, Zaheeruddin, G Singh "Soft Computing Techniques and Role of Power World® Simulator for Forecasting and Mitigation of Power System Blackout," iccima, vol. 1, pp.341-346, 2007. International Conference on Computational Intelligence and Multimedia Applications, 2007. 